\title{
Impact of Aspirin Treatment on Long-Term Outcome (Over 10Years) After Percutaneous Coronary Intervention
}

\author{
Widi NJAMAN, ${ }^{1}$ MD, Katsumi MiYAUCHI, ${ }^{1}$ MD, Takatoshi KASAI, ${ }^{1}$ MD, \\ Takeshi KuRATA, ${ }^{1}$ MD, Hitoshi SATOH, ${ }^{1}$ MD, Hiroshi OHTA, ${ }^{1}$ MD, \\ Shinya OKazAKI, ${ }^{1} \mathrm{MD}$, Ken YокоYAma, ${ }^{1} \mathrm{MD}$, Takahiko KoJIMA, ${ }^{1} \mathrm{MD}$, \\ Yoshinori АкIMOTO, ${ }^{1} \mathrm{MD}$, and Hiroyuki DAIDA, ${ }^{1} \mathrm{MD}$
}

\section{SUMMARY}

Aspirin has been shown to reduce cardiovascular morbidity and mortality following percutaneous coronary intervention (PCI). However, its effects on long-term (over 10years) mortality have not been fully elucidated.

This retrospective study recorded the patient characteristics and admission medication for all patients undergoing PCI over an 8-year period from 1984 to 1992. Follow-up information was available for 748 patients $(100 \%)$ for a mean of $143.6 \pm 43.4$ months. A propensity analysis was performed to adjust for presumed selection biases in the administration of aspirin.

The baseline clinical characteristics were similar between the group that received aspirin and the group that did not, except for the administration of statins and PCI procedural success rate. Of the 748 patients, 535 (71.5\%) received aspirin treatment at the time of PCI. During the 12-year follow-up, 54 patients died from any cause and 20 patients from cardiac death. Kaplan-Meier analysis showed that aspirin treatment led to a significant reduction in all cause mortality $(10 \%$ versus $16.4 \% ; P=0.01)$ and cardiac death $(3.7 \%$ versus $8.0 \% ; P=0.02)$ compared to other antiplatelet drugs. The hazard ratio (HR) for the total mortality and cardiac mortality rates was adjusted using the Cox-proportional hazard model for confounding variables and propensity score. The all cause (HR, 0.49; 95\% CI [0.29-0.80], $P=0.005$ ) and cardiac mortality rates (HR, 0.32; 95\% CI [0.14-0.72], $P=0.006$ ) for patients receiving aspirin remained lower than for those not receiving aspirin.

Aspirin treatment at the time of PCI significantly reduced the risk of death from any cause and cardiac death. The administration of aspirin had a positive impact on the over 10-year long-term outcomes of patients who underwent PCI. (Int Heart J 2006; 47: 37-45)

Key words: Aspirin, Percutaneous coronary intervention, Long-term outcome

From the ${ }^{1}$ Department of Cardiology, Juntendo University School of Medicine, Tokyo, Japan.

Address for correspondence: Katsumi Miyauchi, MD, Department of Cardiology, Juntendo University School of Medicine,

2-1-1 Hongo, Bunkyo-ku, Tokyo 113-8421, Japan.

Received for publication August 4, 2005.

Revised and accepted October 7, 2005. 
ASPIRIN is the most common and effective antiplatelet agent for primary ${ }^{1-3)}$ and secondary ${ }^{4-7)}$ prevention of cardiovascular disease. In a recent meta-analysis, ${ }^{8,9)}$ aspirin was found to reduce the risk of death from any cause, including vascular death, myocardial infarction (MI), and stroke among all patients who were at high risk for vascular events. Moreover, aspirin also decreased the frequency of ischemic complications after PCI. However, there is a paucity of data regarding the long-term effects of aspirin administration on mortality and cardiovascular events in Japanese patients. The present study was designed to investigate whether the administration of aspirin had any effect on the long-term (more than 10 years) clinical outcome associated with PCI.

\section{METHODS}

Patients: The study population consisted of 748 consecutive patients who underwent PCI at our institution between January 1984 and December 1992. Patients were divided into 2 groups according to aspirin usage that had been initiated at the time of PCI. The dose of aspirin administered to these patients was either 81 $\mathrm{mg}$ or $100 \mathrm{mg}$ a day.

Percutaneous coronary intervention (Balloon procedure): Angioplasty was performed using standard techniques and pharmacotherapy. Pharmacotherapy included pretreatment with intravenous heparin (100-150 U/kg bolus injection prior to the procedure and $10 \mathrm{U} / \mathrm{kg} /$ hour after the procedure for 24 hours) and intracoronary nitrates at doses determined by the attending cardiologist during the procedure.

Patient follow-up: The clinical events until September 2002 and the causes of the events were also analyzed. Patient follow-up information was obtained from hospital charts, a questionnaire, and supplemented by telephone interview with the patient or their immediate relatives conducted by a single cardiologist.

The study outcomes were all cause and cardiovascular etiology. Cardiac death was defined as death due to coronary artery disease (CAD), cardiogenic shock (not including congestive heart failure), and sudden death. Cardiovascular events were classified as one of the following: (1) nonfatal acute myocardial infarction, (2) repeat PCI or coronary artery bypass surgery (CABG), or (3) nonfatal cerebral infarction.

Statistical analysis: Continuous data are expressed as the mean \pm SD. The chisquare test was used to compare the baseline characteristics of patients with and without aspirin use, and Student's $t$ test was used for continuous variables. Survival and event-free survival rates were assessed by the Kaplan-Meier method, and survival curves between the 2 groups were compared using the log-rank test. 
The Cox proportional hazard model was used to determine the independent predictors of late mortality. A $P$ value $<0.05$ was considered significant.

To adjust for any bias inherent in the decision about aspirin therapy before PCI, propensity analysis was performed. ${ }^{10,11)}$ Propensity analysis aims to identify patients with a similar probability of receiving aspirin therapy on the basis of observed clinical characteristics. Using a multivariate logistic regression model that includes the baseline characteristics as the independent variables, the probability of being assigned to aspirin therapy was determined. The variables included in the propensity score model were age, gender, BMI, diabetes mellitus, hypertension, smoking, family history of CAD, use of other drugs (nitrates, nicorandil, beta blockers, angiotensin-converting enzyme inhibitors, calcium channel block-

Table I. Baseline Characteristics

\begin{tabular}{|c|c|c|c|}
\hline & $\begin{array}{l}\text { Aspirin users } \\
\quad(n=535)\end{array}$ & $\begin{array}{c}\text { Nonaspirin users } \\
\quad(n=213)\end{array}$ & $P$ value \\
\hline Sex, male/female & $466 / 69$ & $185 / 28$ & NS \\
\hline Mean age (years old) & $59.1 \pm 0.4$ & $59.3 \pm 8.7$ & NS \\
\hline \multicolumn{4}{|l|}{ Coronary risk factors } \\
\hline $\mathrm{BMI}\left(\mathrm{kg} / \mathrm{m}^{2}\right)$ & $23.5 \pm 2.8$ & $23.5 \pm 2.6$ & NS \\
\hline Hypertension & $366(68.4 \%)$ & $141(66.2 \%)$ & NS \\
\hline Diabetes mellitus & $232(43.4 \%)$ & $83(39.0 \%)$ & NS \\
\hline Smoker & $411(76.8 \%)$ & $168(78.8 \%)$ & NS \\
\hline Hyperlipidemia & $455(85.0 \%)$ & $175(82.1 \%)$ & NS \\
\hline Family history of IHD & $186(34.8 \%)$ & $73(34.3 \%)$ & NS \\
\hline \multicolumn{4}{|l|}{ Combined medication } \\
\hline Nitrates & $476(89.0 \%)$ & $196(92.0 \%)$ & NS \\
\hline Nicorandil & $103(19.2 \%)$ & $37(17.3 \%)$ & NS \\
\hline $\mathrm{CCB}$ & $158(29.5 \%)$ & $63(29.6 \%)$ & NS \\
\hline ACE-I & $46(8.6 \%)$ & $12(5.6 \%)$ & NS \\
\hline$\beta$-Blocker & $139(26.0 \%)$ & $47(22.1 \%)$ & NS \\
\hline Statin & $190(35.5 \%)$ & $53(24.9 \%)$ & $<0.01$ \\
\hline Dipyridamole & $352(65.8 \%)$ & $39(18.3 \%)$ & $<0.0001$ \\
\hline Ticlopidine & $36(6.7 \%)$ & $70(32.9 \%)$ & $<0.0001$ \\
\hline Warfarin & $184(34.4 \%)$ & $106(49.8 \%)$ & 0.0001 \\
\hline \multicolumn{4}{|l|}{ Procedure } \\
\hline ACS & $148(27.6 \%)$ & $68(31.9 \%)$ & NS \\
\hline History of PCI & $149(27.8 \%)$ & $49(23.0 \%)$ & NS \\
\hline History of CABG & $100(18.6 \%)$ & $35(16.4 \%)$ & NS \\
\hline $\operatorname{LVEF}(\%)$ & $67.3 \pm 11.7$ & $65.8 \pm 11.0$ & NS \\
\hline Number of diseased coronary arteries & $1.7 \pm 0.6$ & $1.5 \pm 0.5$ & 0.02 \\
\hline LMT lesion & $8(1.4 \%)$ & $3(1.4 \%)$ & NS \\
\hline LAD lesion & $274(51.2 \%)$ & $109(51.1 \%)$ & NS \\
\hline SVG lesion & $48(8.9 \%)$ & $16(7.5 \%)$ & NS \\
\hline Procedural success $(\%)$ & $478(89.3 \%)$ & $175(82.1 \%)$ & 0.01 \\
\hline
\end{tabular}

BMI indicates body mass index; IHD, ischemic heart disease; CCB, calcium channel blocker; ACE-I, angiotensin-converting enzyme inhibitor; ASC, acute coronary syndrome; PCI, percutaneous coronary intervention; CABG, coronary artery bypass graft; LVEF, left ventricular ejection fraction; LMT, left main tract; LAD, left anterior descending; and SVG, saphenous vein graft. 
ers, statins, and other antithrombotic agents), presence of acute coronary syndrome, history of previous PCI and/or CABG, left ventricular ejection fraction (LVEF), number of diseased coronary arteries, left main tract (LMT), left anterior descending (LAD), or saphenous vein graft (SVG) lesion, procedural success (defined as a residual stenosis $<50 \%$ after PCI), and propensity score. This score was then incorporated into subsequent proportional-hazard models as a covariate.

\section{RESULTS}

Patient characteristics: The baseline characteristics of the 748 patients are summarized in Table I. Aspirin was taken by 535 patients (71.5\%) while 213 patients $(28.5 \%)$ received other antiplatelet or anticoagulant medication. There were no significant differences in age, gender, BMI, or the presence of coronary risk factors between the 2 groups. Patients treated with aspirin received more statin therapy, and were more likely to have multivessel disease and significantly higher success rates than patients not using aspirin. With respect to coadministration with other antiplatelet or anticoagulant agents, aspirin users took dipyridamole more often than nonaspirin users, but they received less ticlopidine and warfarin. Long-term outcome: During the follow-up period, 89 patients died, 37 of whom died due to a cardiac-related cause. There was a $6.4 \%$ reduction in all causes of mortality in patients undergoing aspirin therapy (10\% versus $16.4 \%, P=0.02)$. Similarly, the cardiac death rate was $4.2 \%$ lower in patients with aspirin than patients without aspirin $(3.7 \%$ versus $17 \%, P=0.02)$ (Table II). Kaplan-Meier analysis demonstrated a significant reduction in all causes of death for aspirin users (log-rank $P=0.01)$. Cardiac mortality was significantly lower in patients

Table II. Long-term Outcome for Patients Taking and not Taking Aspirin

\begin{tabular}{lccc}
\hline & $\begin{array}{c}\text { Aspirin users } \\
(n=535)\end{array}$ & $\begin{array}{c}\text { Nonaspirin users } \\
(n=213)\end{array}$ & $P$ value \\
\hline Mean follow-up period (months) & $141.6 \pm 43.3$ & $142.8 \pm 55.0$ & NS \\
All cause of death & $54(10 \%)$ & $35(16.4 \%)$ & 0.02 \\
Cardiac death & $20(3.7 \%)$ & $17(7.9 \%)$ & 0.02 \\
Noncardiac death & $34(6.3 \%)$ & $18(8.4 \%)$ & NS \\
Stroke death & $5(0.9 \%)$ & $5(2.3 \%)$ & NS \\
Death by bleeding & $2(0.3 \%)$ & $1(0.4 \%)$ & NS \\
Cardiovascular event & $239(44.6 \%)$ & $97(45.5 \%)$ & NS \\
AMI & $25(4.6 \%)$ & $9(4.2 \%)$ & NS \\
CABG & $58(10.8 \%)$ & $26(12.2 \%)$ & NS \\
Repeat PCI & $183(34.2 \%)$ & $63(29.5 \%)$ & NS \\
TVR & $146(27.2 \%)$ & $46(21.5 \%)$ & NS \\
New lesion & $85(15.8 \%)$ & $37(17.3 \%)$ & NS \\
Cerebral infarction & $18(8.3 \%)$ & $10(4.6 \%)$ & NS \\
\hline
\end{tabular}

TVR indicates target vessel revascularization. 

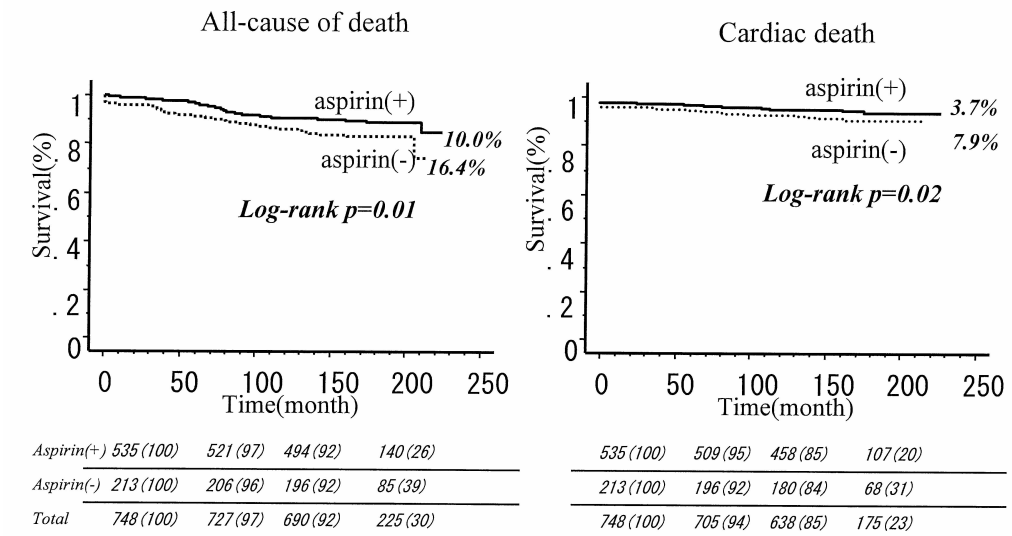

Figure. Kaplan-Meier curve of survival according to aspirin therapy after PCI. The left shows all-cause of death free survival and the right shows cardiac death free survival.

Table III. Cox Regression Unadjusted and Adjusted Analysis of Endpoints

\begin{tabular}{lcccc}
\hline & \multicolumn{2}{c}{ Unadjusted analysis } & \multicolumn{2}{c}{ Adjusted analysis } \\
& HR $[95 \% \mathrm{CI}]$ & $P$ value & HR $[95 \% \mathrm{CI}]$ & $P$ value \\
\hline All cause death & $0.59[0.38-0.91]$ & 0.01 & $0.49[0.29-0.80]$ & 0.005 \\
Cardiac death & $0.47[0.24-0.91]$ & 0.02 & $0.32[0.14-0.72]$ & 0.006 \\
Noncardiac death & $0.88[0.49-1.57]$ & NS & $0.90[0.53-1.52]$ & NS \\
Cardiac events & $1.02[0.80-1.29]$ & NS & $1.07[0.82-1.39]$ & NS \\
\hline
\end{tabular}

Covariates: age, gender, BMI, diabetes mellitus, hypertension, smoking, family history of IHD, use of other drugs (nitrate, nicorandil, beta blocker, ACE-I, CCB, statin, other antithrombotic agents), presence of ACS, history of previous PCI and/or CABG, LVEF, number of disease coronary arteries, presence of LMT/LAD/ SVG lesion, procedural success (defined as a residual stenosis $<50 \%$ after PCI), and propensity score.

taking aspirin $(\log$ rank $P=0.02)$ than in those not taking aspirin, and the reduction was similar to that seen for all causes of mortality (Figure).

The hazard ratios (HR) for the endpoint for the aspirin users compared with the nonaspirin users are presented in Table III. After adjusting for the derived propensity score, the benefit of aspirin remained unchanged. The risks of all causes of death $(\mathrm{HR}=0.49,95 \% \mathrm{CI}[0.29-0.80], P=0.005)$ and cardiac death $(\mathrm{HR}=$ $0.32,95 \%$ CI [0.14-0.72], $P=0.006)$ were significantly lower in aspirin users (Table III).

There were no significant differences in all causes of mortality and cardiac mortality compared with aspirin treatment alone and coadministration with other antiplatelet or anticoagulant agents (data not shown). 


\section{DISCUSSION}

On the basis of our registry data, aspirin treatment at the time of PCI is associated with a reduction in long-term mortality, defined here as being over 10 years. This was a retrospective and observational study and used propensity analysis to adjust for the different backgrounds. The results indicated aspirin therapy has long-term efficacy in Japanese PCI patients.

Several reports have described the benefits of aspirin treatment in PCI patients. Schwartz, et $a l^{12)}$ reported in an early study of 376 patients undergoing angioplasty and randomized to receive either aspirin $(990 \mathrm{mg}$ ) and dipyridamole ( $225 \mathrm{mg}$ daily) or placebo for 4 to 7 months after angioplasty that there was a reduction in periprocedural Q-wave $\mathrm{MI}$ when antiplatelet therapy was started before the procedure $(6.9 \%$ versus $1.6 \% ; P=0.011)$. In a randomized trial of long-term aspirin therapy following angioplasty, Savage, et al ${ }^{13)}$ reported that aspirin (325 mg daily) given for 6 months was associated with a significant reduction in $\mathrm{MI}$ compared with placebo $(1.2 \%$ versus $5.7 \% ; P=0.03)$.

The mortality benefits of aspirin treatment after PCI have also been observed in meta-analysis. The Antithrombotic Trialists' Collaboration overview $^{8)}$ showed that there was a $17 \%$ reduction in death from any cause and a $15 \%$ reduction in cardiac death among patients who were at high risk for vascular events. Among a subset of post-PCI patients, there was a 53\% reduction in vascular events (nonfatal MI, nonfatal stroke, or death from a vascular cause).

The immediate and short-term outcomes of PCI with aspirin treatment have been investigated in Japanese populations whose cardiovascular mortality and rate of bleeding complications were different from those in Western populations, however, there was little consideration of the impact of aspirin treatment on longterm outcome. ${ }^{14,15)}$ In a randomized study that evaluated the efficacy of aspirin at the dose of $81 \mathrm{mg} /$ day in 723 AMI patients, ${ }^{14)}$ long-term aspirin use for a mean of 475 days reduced the incidence of recurrent AMI compared with the group receiving no antiplatelet agent after AMI $(0.02 \%$ versus $0.07 \%, P=0.0045)$. However, there were no differences in the incidences of cardiovascular events that included cardiovascular death, reinfarction, uncontrolled unstable angina requiring admission to hospital, and nonfatal ischemic stroke.

Aspirin inhibits platelet cyclooxygenase by irreversible acetylation, thereby preventing the formation of thromboxane A2. ${ }^{16,17)}$ In addition to its antiplatelet effect, aspirin has anti-inflammatory activity that may contribute to its clinical effectiveness in preventing serious vascular events, and also reduces the frequency of ischemic complication after PCI. ${ }^{12,13,18,19)}$ Moreover, in experimental studies, other mechanisms have been proposed to explain the long-term reduction in mortality due to coronary artery disease. One of the mechanisms is that aspirin 
improves endothelial dysfunction in artherosclerotic vessels. Aspirin helps to enhance NO production in endothelial cells by inhibiting prostacyclin synthesis, which appears to be mediated by a nitric oxide (NO)/cGMP-dependent process ${ }^{20}$ and inhibiting platelet activation by neutrophils. ${ }^{21)}$ Other available data have suggested that aspirin plays an important role as an antioxidant. Aspirin has been shown to scavenge hydroxyl radicals to form the 2,3- and 2,5-dihydroxybenzoate derivatives, which are recognized as markers of oxidative stress. ${ }^{22}$ A recent study has demonstrated that aspirin elicits NO release from vascular endothelium as well as an increase in eNOS activity in endothelial cell homogenates. ${ }^{23)}$

A meta-analysis study ${ }^{8)}$ reported it is difficult to evaluate the long-term outcome of aspirin therapy because there are no large directly randomized comparisons of different durations of treatment. Although the present data were obtained at a single institution, we were able to demonstrate that long-term aspirin therapy had the potential to make a major impact with respect to mortality.

Analysis of the baseline characteristics of the patients showed that there were more who took both aspirin and an HMG-CoA reductase inhibitor (or statin) than those who took a statin alone $(35.5 \%$ versus $24.9 \%, P<0.01)$. Furthermore, patients who were administered aspirin had a higher procedural success rate $(89.3 \%$ versus $82.1 \%, P=0.01)$ and a greater number of diseased coronary arteries $(1.7 \pm 0.6$ versus $1.5 \pm 0.5, P=0.02)$. Secondary prevention trials with statins have shown a $25 \%$ to $30 \%$ reduction in ischemic cardiovascular events. ${ }^{24-26)}$ In the present study, aspirin treatment was effective at reducing the rate of all cause death $(\mathrm{HR}=0.62,95 \%$ CI $[0.40-0.94], P=0.02)$ and cardiac death $(\mathrm{HR}=0.49$, 95\%CI [0.25-0.96], $P<0.05)$ when patients who took statins were excluded. Thus, the results of the present study were able to demonstrate the efficacy of long-term aspirin treatment after PCI despite the influence of statin coadministration. In patients who underwent unsuccessful PCI, there was no statistically significant difference in the efficacy of aspirin therapy in terms of mortality (HR = $0.59,95 \%$ CI [0.22-1.59], $P=0.30)$. However, the fact that the procedural success rate had no influence on the beneficial effect of aspirin treatment with regards to long-term outcome may be explained as follows. First, it was difficult to evaluate the group excluding patients who underwent successful PCI because of its small population size $(n=95)$. Second, multivariate analysis showed aspirin was a strong independent predictor in our study.

Study limitations: This study has several limitations. First, it is a single center, observational study of daily clinical practice with retrospective data collection. Although the propensity score is a powerful and acceptable methodology in statistical analysis, ${ }^{27)}$ there are inherent limitations in selection and referral bias. Moreover, further investigation is needed to evaluate data from other institutions with different operators and various PCI devices. 
Second, the compliance with aspirin treatment throughout the follow-up period was not determined. In this study population, $35 \%$ of patients who were not on aspirin therapy at discharge subsequently started to take aspirin and $15 \%$ of patients who took aspirin at discharge ceased therapy. Crossover between aspirin users and nonusers would lead to underestimation of the therapeutic efficacy, and it may strengthen our conclusion about the beneficial effects of aspirin at the time of PCI.

Third, the entire study population underwent coronary balloon angioplasty. It has been widely established that coronary stenting therapy has a superior angiographic clinical outcome than that of balloon angioplasty. ${ }^{28,29)}$ The results of longterm (over 10 years) follow-up analysis should be awaited in order to evaluate the efficacy of aspirin treatment after various PCI procedures, including drug-eluting stents. Despite these limitations, this study represents a large cohort of patients with complete long-term clinical follow-up.

Conclusion: In a retrospective study involving 748 consecutive PCI patients, we have demonstrated that the use of aspirin significantly reduces long-term all cause and cardiovascular mortality (over 10 years) after PCI. Although the efficacy of aspirin treatment exceeding 10 years has not been adequately evaluated, the present results seem to indicate there is a need to continue post-PCI aspirin administration.

\section{REFERENCES}

1. Peto R, Gray R, Collins R, et al. Randomised trial of prophylactic daily aspirin in British male doctors. Br Med J (Clin Res Ed) 1988; 296: 313-6.

2. Physician's health study: aspirin and primary prevention of coronary heart disease. N Engl J Med 1989; 321: 1825-8.

3. Manson JE, Stampfer MJ, Colditz GA, et al. A prospective study of aspirin use and primary prevention of cardiovascular disease in women. JAMA 1991; 266: 521-7.

4. Fuster V, Dyken ML, Vokonas PS, Hennekens C. Aspirin as a therapeutic agent in cardiovascular disease. Special Writing Group. Circulation 1993; 87: 659-75. (Review)

5. Hennekens CH, Buring JE, Standercock P, Collins R, Peto R. Aspirin and other antiplatelet agents in the secondary and primary prevention of cardiovascular disease. Circulation 1989; 80: 749-56. (Review)

6. Secondary prevention of vascular disease by prolonged antiplatelet treatment. Antiplatelet Trialists' Collaboration. Br Med J (Clin Res Ed) 1988; 296: 320-31.

7. Juul-Moller S, Edvardsson N, Jahnmatz B, Rosen A, Sorensen S, Omblus R. Double-blind trial of aspirin in primary prevention of myocardial infarction in patients with stable chronic angina pectoris. The Swedish Angina Pectoris Aspirin Trial (SAPAT) Group. Lancet 1992; 340: 1421-5.

8. Collaborative overview of randomised trials of antiplatelet therapy--I: Prevention of death, myocardial infarction, and stroke by prolonged antiplatelet therapy in various categories of patients. Antiplatelet Trialists' Collaboration. BMJ 1994; 308: 81-106.

9. Antithrombotic Trialists' Collaboration. Collaborative meta-analysis of randomised trials of antiplatelet therapy for prevention of death, myocardial infarction, and stroke in high risk patients. BMJ 2002; 324: 71-86. 
10. Brener SJ, Lytle BW, Casserly IP, Schneider JP, Topol EJ, Lauer MS. Propensity analysis of long-term survival after surgical or percutaneous revascularization in patients with multivessel coronary artery disease and highrisk features. Circulation 2004; 109: 2290-5.

11. Chan AW, Bhatt DL, Chew DP, et al. Early and sustained survival benefit associated with statin therapy at the time of percutaneous coronary intervention. Circulation 2002; 105: 691-6.

12. Schwartz L, Bourassa MG, Lesperance J, et al. Aspirin and dipyridamole in the prevention of restenosis after percutaneous transluminal coronary angioplasty. N Engl J Med 1988; 318: 1714-9.

13. Savage MP, Goldberg S, Bove AA, et al. Effect of thromboxane A2 blockade on clinical outcome and restenosis after successful coronary angioplasty. Multi-Hospital Eastern Atlantic Restenosis Trial (M-HEART II). Circulation 1995; 92: 3194-200.

14. Yasue H, Ogawa $\mathrm{H}$, Tanaka $\mathrm{H}$, et al. Effects of aspirin and trapidil on cardiovascular events after acute myocardial infarction. Japanese Antiplatelets Myocardial Infarction Study (JAMIS) Investigators. Am J Cardiol 1999; 83: 1308-13.

15. Kawata M, Kuramoto E, Kataoka T, et al. Comparative inhibitory effects of cilostazol and ticlopidine on subacute stent thrombosis and platelet function in acute myocardial infarction patients with percutaneous coronary intervention. Int Heart J 2005; 46:13-22.

16. Awtry EH, Loscalzo J. Aspirin. Circulation 2000; 101: 1206-18.

17. Vane JR. Inhibition of prostaglandin synthesis as a mechanism of action for aspirin-like drugs. Nat New Biol 1971; 231: 232-5.

18. Lembo NJ, Black AJ, Roubin GS, et al. Effect of pretreatment with aspirin versus aspirin plus dipyridamole on frequency and type of acute complications of percutaneous transluminal coronary angioplasty. Am J Cardiol 1990; 65: 422-6.

19. Knudtson ML, Flintoft VF, Roth DL, Hansen JL, Duff HJ. Effect of short-term prostacyclin administration on restenosis after percutaneous transluminal coronary angioplasty. J Am Coll Cardiol 1990; 15: 691-7.

20. Bolz SS, Pohl U. Indomethacin enhances endothelial NO release--evidence for a role of PGI2 in the autocrine control of calcium-dependent autacoid production. Cardiovasc Res 1997; 36: 437-44.

21. De La Cruz, JP, Blanco E, Sanchez de la Cuesta F. Effect of dipyridamole and aspirin on the platelet-neutrophil interaction via the nitric oxide pathway. Eur J Pharmacol 2000; 397: 35-41.

22. Ghiselli A, Laurenti O, De Mattia G, Maiani G, Furro-Luzzi A. Salicylate hydroxylation as an early marker of in vivo oxidative stress in diabetic patients. Free Radic Biol Med 1992; 13: 621-6.

23. Taubert D, Berkels R, Grosser N, Schroder H, Grundemann D, Schoming E. Aspirin induces nitric oxide release from vascular endothelium: a novel mechanism of action. Br J Pharmacol 2004; 143: 159-65.

24. Randomised trial of cholesterol lowering in 4444 patients with coronary heart disease: the Scandinavian Simvastatin Survival Study (4S). Lancet 1994; 344: 1383-9.

25. Sacks FM, Pfeffer MA, Moya LA, et al. The effect of pravastatin on coronary events after myocardial infarction in patients with average cholesterol levels. Cholesterol and Recurrent Events Trial investigators. N Engl J Med 1996; 335: 1001-9.

26. Prevention of cardiovascular events and death with pravastatin in patients with coronary heart disease and a broad range of initial cholesterol levels. The Long-Term Intervention with Pravastatin in Ischaemic Disease (LIPID) Study Group. N Engl J Med 1998; 339: 1349-57.

27. Radford MJ, Foody JM. How do observational studies expand the evidence base for therapy? JAMA 2001; 286: 1228-30.

28. Fischman DL, Leon MB, Baim DS, et al. A randomized comparison of coronary-stent placement and balloon angioplasty in the treatment of coronary artery disease. Stent Restenosis Study Investigators. N Engl J Med 1994; 331: 496-501.

29. Serruys PW, de Jaegere P, Kiemeneij F, et al. A comparison of balloon-expandable-stent implantation with balloon angioplasty in patients with coronary artery disease. Benestent Study Group. N Engl J Med 1994; 331 : 489-95. 